\title{
The Triangular Hermite Finite Element Complementing the Bogner-Fox-Schmit Rectangle
}

\author{
Lidia Gileva $^{1}$, Vladimir Shaydurov ${ }^{1,2}$, Boris Dobronets ${ }^{3}$ \\ ${ }^{1}$ Institute of Computational Modeling, Siberian Branch of Russian Academy of Sciences, Krasnoyarsk, Russia \\ ${ }^{2}$ Beihang University, Beijing, China \\ ${ }^{3}$ Siberian Federal University, Krasnoyarsk, Russia \\ Email: shaidurov04@mail.ru
}

Received October 20, 2013; revised November 20, 2013; accepted November 27, 2013

Copyright (C) 2013 Lidia Gileva et al. This is an open access article distributed under the Creative Commons Attribution License, which permits unrestricted use, distribution, and reproduction in any medium, provided the original work is properly cited. In accordance of the Creative Commons Attribution License all Copyrights (C) 2013 are reserved for SCIRP and the owner of the intellectual property Lidia Gileva et al. All Copyright (C) 2013 are guarded by law and by SCIRP as a guardian.

\begin{abstract}
The Bogner-Fox-Schmit rectangular element is one of the simplest elements that provide continuous differentiability of an approximate solution in the framework of the finite element method. However, it can be applied only on a simple domain composed of rectangles or parallelograms whose sides are parallel to two different straight lines. We propose a new triangular Hermite element with 13 degrees of freedom. It is used in combination with the Bogner-Fox-Schmit element near the boundary of an arbitrary polygonal domain and provides continuous differentiability of an approximate solution in the whole domain up to the boundary.
\end{abstract}

Keywords: Continuously Differentiable Finite Elements; Bogner-Fox-Schmit Rectangle; Triangular Hermite Element

\section{Introduction}

The finite elements with inter-elemental continuous differentiability are more complicated than those providing only continuity. Such two-dimensional elements are mostly developed for triangles: the Argyris triangle [1], the Bell reduced triangle [2], the family of Morgan-Scott triangles [3], the Hsieh-Clough-Tocher macrotriangle [4], the reduced Hsieh-Clough-Tocher macrotriangle [5], the family of Douglas-Dupont-Percell-Scott triangles [6], and the Powell-Sabin macrotriangles [7]. The Fraeijs de Veubeke-Sander quadrilateral [8] and its reduced version [9] are also composed of triangles. As for single, noncomposite rectangles, the Bogner-Fox-Schmit (BFS) element [10] is the most popular and simplest one in the family of elements discribed by Zhang [11]. All these elements are widely used in the conforming finite element method for the biharmonic equation and other equations of the fourth order (see for example [12-18] and references therein) along with mixed statements of problems and a nonconforming approach $[12,14,17]$.

A direct application of the BFS-element is restricted to the case of a simple domain that is composed of rectangles or parallelograms whose sides are parallel to two different straight lines. This condition fails even in the case of a simple polygonal domain where the intersection of the boundary with rectangles results in triangular cells (cf. Figure 1). Of course, one can construct the special triangulation compatible with the boundary as some isoparametric image [19] of a domain composed of rectangles with sides parallel to the axes. This way requires solving some additional boundary value problems for the construction of such a mapping that is smooth over the whole domain.

In this paper we suggest to use the BFS-elements in the direct way (without an isoparametric mapping) for a couple with the proposed triangular Hermite elements with 13 degrees of freedom. These triangular elements supplement BFS-elements in the following sense. They are used only near the boundary of a polygonal domain and provide inter-element continuous differentiability between finite elements of these two types. Thus, due to the joint use of these elements on a polygonal domain $\Omega$, the approximate solution of finite element method belongs to the class $C^{1}(\bar{\Omega})$ of functions which are continuously differentiable on the closure $\bar{\Omega}$ of the domain. 


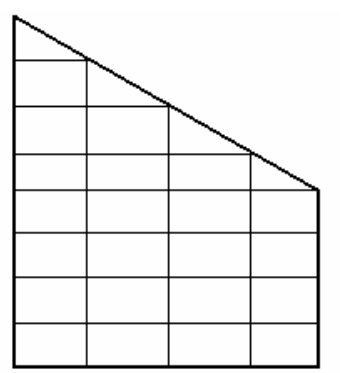

Figure 1. A subdivision of a domain.

\section{Triangulation of a Domain and the Bogner-Fox-Schmit Element}

Let $\Omega \subset R^{2}$ be a convex polygon with a boundary $\Gamma$. Assume that we can construct a triangulation $\mathfrak{J}_{h}$ of $\Omega$ subdividing it into closed rectangular and triangular cells so that the most of them are rectangles and only a part of the cells adjacent to the boundary may be rectangular triangles. Besides, any two cells of $\mathfrak{J}_{h}$ may not have a common interior point and any two triangular cells may not have a common side. In addition, the union of all the cells coincides with $\bar{\Omega}$. A simple example of the triangulation is shown in Figure 1. We also assume that all sides of the rectangular cells and the catheti of the triangular cells are parallel to the axes.

Denote by $h$ the maximal diameter of all meshes $e \in \mathfrak{J}_{h}$. On rectangular cells we use the Bogner-FoxSchmit element [1]. It is defined by the triple $\left(e, P_{e}^{1}, \Sigma_{e}^{1}\right)$ where $e$ is the rectangle with vertices $a_{1}^{e}=\left(a_{1,1}^{e}, a_{1,2}^{e}\right)$, $a_{2}^{e}=\left(a_{2,1}^{e}, a_{1,2}^{e}\right), \quad a_{3}^{e}=\left(a_{2,1}^{e}, a_{4,2}^{e}\right), \quad$ and $\quad a_{4}^{e}=\left(a_{1,1}^{e}, a_{4,2}^{e}\right)$ and with side lengths $h_{1}^{e}=a_{2,1}^{e}-a_{1,1}^{e}$ and $h_{2}^{e}=a_{4,2}^{e}-a_{1,2}^{e}$. Besides, $P_{e}^{1}=Q_{3}(e)$ is the space of bicubic polynomials on $e$; and $\Sigma_{e}^{1}$ is the set of linear functionals (degrees of freedom or nodal parameters) of the form [12]

$$
\begin{aligned}
& \Sigma_{e}^{1}=\left\{\psi_{i, 1}(p)=p\left(a_{i}^{e}\right), \psi_{i, 2}(p)=\partial_{1} p\left(a_{i}^{e}\right),\right. \\
& \psi_{i, 3}(p)=\partial_{2} p\left(a_{i}^{e}\right), \psi_{i, 4}(p)=\partial_{1,2} p\left(a_{i}^{e}\right), \\
& \left.i=1, \cdots, 4, p \in P_{e}^{1}\right\} .
\end{aligned}
$$

The dimension of $P_{e}^{1}=Q_{3}(e)$ (the number of the coefficients of a polynomial) is equal to 16 and coincides with the number of the degrees of freedom. For this element we have the Lagrange basis in $P_{e}^{1}$ that consists of the functions $\varphi_{i, j}^{e}(x) \quad(i, j=1, \cdots, 4)$ satisfying the condition

$$
\psi_{i, j}^{e}\left(\varphi_{k, l}^{e}\right)=\delta_{i, k} \delta_{j, l} \text { for } i, j, k, l=1, \cdots, 4,
$$

where $\delta$ is the Kronecker delta. These functions can be written in the explicit form with the help of the one-dimensional splines

$$
\hat{\phi}_{0}(x)=1-3 x^{2}+2 x^{3} \text { and } \hat{\phi}_{1}(x)=x-2 x^{2}+x^{3} \text {. }
$$

Indeed, the direct calculations show that the basis has the form

$$
\begin{aligned}
& \varphi_{1,1}^{e}=\hat{\varphi}_{0}\left(\left(x-a_{1,1}^{e}\right) / h_{1}^{e}\right) \hat{\varphi}_{0}\left(\left(y-a_{1,2}^{e}\right) / h_{2}^{e}\right), \\
& \varphi_{1,2}^{e}=\hat{\varphi}_{1}\left(\left(x-a_{1,1}^{e}\right) / h_{1}^{e}\right) \hat{\varphi}_{0}\left(\left(y-a_{1,2}^{e}\right) / h_{2}^{e}\right), \\
& \varphi_{1,3}^{e}=\hat{\varphi}_{0}\left(\left(x-a_{1,1}^{e}\right) / h_{1}^{e}\right) \hat{\varphi}_{1}\left(\left(y-a_{1,2}^{e}\right) / h_{2}^{e}\right), \\
& \varphi_{1,4}^{e}=\hat{\varphi}_{1}\left(\left(x-a_{1,1}^{e}\right) / h_{1}^{e}\right) \hat{\varphi}_{1}\left(\left(y-a_{1,2}^{e}\right) / h_{2}^{e}\right), \\
& \varphi_{2,1}^{e}=\hat{\varphi}_{0}\left(\left(a_{2,1}^{e}-x\right) / h_{1}^{e}\right) \hat{\varphi}_{0}\left(\left(y-a_{1,2}^{e}\right) / h_{2}^{e}\right), \\
& \varphi_{2,2}^{e}=\hat{\varphi}_{1}\left(\left(a_{2,1}^{e}-x\right) / h_{1}^{e}\right) \hat{\varphi}_{0}\left(\left(y-a_{1,2}^{e}\right) / h_{2}^{e}\right), \\
& \varphi_{2,3}^{e}=\hat{\varphi}_{0}\left(\left(a_{2,1}^{e}-x\right) / h_{1}^{e}\right) \hat{\varphi}_{1}\left(\left(y-a_{1,2}^{e}\right) / h_{2}^{e}\right), \\
& \varphi_{2,4}^{e}=\hat{\varphi}_{1}\left(\left(a_{2,1}^{e}-x\right) / h_{1}^{e}\right) \hat{\varphi}_{1}\left(\left(y-a_{1,2}^{e}\right) / h_{2}^{e}\right), \\
& \varphi_{3,1}^{e}=\hat{\varphi}_{0}\left(\left(x-a_{1,1}^{e}\right) / h_{1}^{e}\right) \hat{\varphi}_{0}\left(\left(a_{4,2}^{e}-y\right) / h_{2}^{e}\right), \\
& \varphi_{3,2}^{e}=\hat{\varphi}_{1}\left(\left(x-a_{1,1}^{e}\right) / h_{1}^{e}\right) \hat{\varphi}_{0}\left(\left(a_{4,2}^{e}-y\right) / h_{2}^{e}\right), \\
& \varphi_{3,3}^{e}=\hat{\varphi}_{0}\left(\left(x-a_{1,1}^{e}\right) / h_{1}^{e}\right) \hat{\varphi}_{1}\left(\left(a_{4,2}^{e}-y\right) / h_{2}^{e}\right), \\
& \varphi_{4,3}^{e}=\hat{\varphi}_{0}\left(\left(a_{2,1}^{e}-x\right) / h_{1}^{e}\right) \hat{\varphi}_{1}\left(\left(a_{4,2}^{e}-y\right) / h_{2}^{e}\right), \\
& \left.\left.\left.\varphi_{4,1}^{e}=\hat{\varphi}_{0}\left(\left(a_{2,1}^{e}-x\right) / h_{1}^{e}\right) \hat{\varphi}_{0}\left(\left(a_{4,2}^{e}-y\right) / h_{2}^{e}\right), h_{1}^{e}\right) \hat{\varphi}_{0}\left(\left(a_{4,2}^{e}-y\right) / h_{2}^{e}\right), x\right) / h_{1}^{e}\right) \hat{\varphi}_{1}\left(\left(a_{4,2}^{e}-y\right) / h_{2}^{e}\right) . \\
& \left.\left.\varphi_{1,1}^{e}\right) / h_{1}^{e}\right) \hat{\varphi}_{1}\left(\left(a_{4,2}^{e}-y\right) / h_{2}^{e}\right), \\
& \left.\varphi_{1,1}^{e}-x\right)
\end{aligned}
$$

\section{The "Reference" Triangular Hermite Element}

First we construct the "reference" triangular element $\left(\hat{e}, P_{\hat{e}}^{2}, \Sigma_{\hat{e}}^{2}\right)$ with the specified properties. We consider the right triangle $\hat{e}$ which has 4 nodes $\hat{a}_{i}, i=1, \cdots, 4$, with the coordinates $(0,0),(1,0),(0.5,0.5)$, and $(0,1)$, respectively (Figure 2).

We define the space $P_{\hat{e}}^{2}$ of functions and the set $\Sigma_{\hat{e}}^{2}$ of degrees of freedom as follows:

$$
\begin{gathered}
P_{\hat{e}}^{2}=\operatorname{span}\left\{1, \hat{x}_{1}, \hat{x}_{2}, \hat{x}_{1}^{2}, \hat{x}_{2}^{2}, \hat{x}_{1} \hat{x}_{2}, \hat{x}_{1}^{3}, \hat{x}_{2}^{3},\right. \\
\left.\hat{x}_{1}^{2} \hat{x}_{2}, \hat{x}_{1} \hat{x}_{2}^{2}, \hat{x}_{1}^{2} \hat{x}_{2}^{2}, \hat{x}_{1}^{3} \hat{x}_{2}, \hat{x}_{1} \hat{x}_{2}^{3}\right\}, \\
\sum_{\hat{e}}^{2}=\left\{\hat{\psi}_{i, 1}(\hat{p})=\hat{p}\left(\hat{a}_{i}\right), i=1, \cdots, 4,\right. \\
\hat{\psi}_{i, 2}(\hat{p})=\partial_{1} \hat{p}\left(\hat{a}_{i}\right), \hat{\psi}_{i, 3}(\hat{p})=\partial_{2} \hat{p}\left(\hat{a}_{i}\right), \\
\left.\hat{\psi}_{i, 4}(\hat{p})=\partial_{1,2} \hat{p}\left(\hat{a}_{i}\right), i=1,2,4, \hat{p} \in P_{\hat{e}}^{2}\right\} .
\end{gathered}
$$




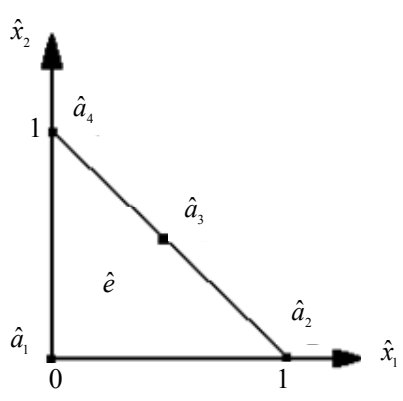

Figure 2. The "reference" triangular element.

Observe that at each of the nodes $\hat{a}_{1}, \hat{a}_{2}$, and $\hat{a}_{4}$ there are 4 degrees of freedom and at the node $\hat{a}_{3}$ there is only one degree. Besides, the degrees of freedom for the nodes $\hat{a}_{1}, \hat{a}_{2}$, and $\hat{a}_{4}$ coincide with those for the nodes of the BFS-element (1).

Lemma 1. The triple $\left(\hat{e}, P_{\hat{e}}^{2}, \Sigma_{\hat{e}}^{2}\right)$ is a finite element.

Proof. The dimension of the space $P_{\hat{e}}^{2}$ coincides with the number of elements of the set $\Sigma_{\hat{e}}^{2}$. Hence, to prove unisolvence of the couple $\left(P_{\hat{e}}^{2}, \Sigma_{\hat{e}}^{2}\right)$, it is sufficient to construct the Lagrange basis $\left\{\hat{\varphi}_{i, j}\left(\hat{x}_{1}, \hat{x}_{2}\right) \in P_{\hat{e}}^{2}\right.$, where $j=1, \cdots, 4$ for $i=1,2,4$ and $j=1$ for $i=3\}$ on $\hat{e}$ satisfying the condition [12]

$$
\hat{\psi}_{i, j}\left(\hat{\varphi}_{k, l}\right)=\delta_{i, k} \delta_{j, l} .
$$

The direct calculations show that the Lagrange basis has the following form:

$$
\begin{aligned}
& \hat{\varphi}_{1,1}=1-3 \hat{x}_{1}^{2}+2 \hat{x}_{1}^{3}-3 \hat{x}_{2}^{2}+2 \hat{x}_{2}^{3}, \\
& \hat{\varphi}_{1,2}=\hat{x}_{1}-2 \hat{x}_{1}^{2}+\hat{x}_{1}^{3}-3 \hat{x}_{1} \hat{x}_{2}^{2}+2 \hat{x}_{1} \hat{x}_{2}^{3}+2 \hat{x}_{1}^{2} \hat{x}_{2}^{2}, \\
& \hat{\varphi}_{1,3}=\hat{x}_{2}-3 \hat{x}_{1}^{2} \hat{x}_{2}+2 \hat{x}_{1}^{3} \hat{x}_{2}-2 \hat{x}_{2}^{2}+\hat{x}_{2}^{3}+2 \hat{x}_{1}^{2} \hat{x}_{2}^{2}, \\
& \hat{\varphi}_{1,4}=\hat{x}_{1} \hat{x}_{2}-2 \hat{x}_{1}^{2} \hat{x}_{2}+\hat{x}_{1}^{3} \hat{x}_{2}-2 \hat{x}_{1} \hat{x}_{2}^{2}+\hat{x}_{1} \hat{x}_{2}^{3}+2 \hat{x}_{1}^{2} \hat{x}_{2}^{2}, \\
& \hat{\varphi}_{2,2}=-\hat{x}_{1}^{2}+\hat{x}_{1}^{3}+2 \hat{x}_{1}^{2} \hat{x}_{2}^{2}, \\
& \hat{\varphi}_{2,3}=3 \hat{x}_{1}^{2} \hat{x}_{2}-2 \hat{x}_{1}^{3} \hat{x}_{2}-4 \hat{x}_{1}^{2} \hat{x}_{2}^{2}, \\
& \hat{\varphi}_{2,4}=-\hat{x}_{1}^{2} \hat{x}_{2}+\hat{x}_{1}^{3} \hat{x}_{2}+\hat{x}_{1}^{2} \hat{x}_{2}^{2}, \\
& \hat{\varphi}_{3,1}=16 \hat{x}_{1}^{2} \hat{x}_{2}^{2} \\
& \hat{\varphi}_{4,1}=3 \hat{x}_{2}^{2}-2 \hat{x}_{2}^{3}-18 \hat{x}_{1}^{2} \hat{x}_{2}^{2}, \\
& \hat{\varphi}_{4,2}=-\hat{x}_{2}^{2}+\hat{x}_{2}^{3}+2 \hat{x}_{1}^{2} \hat{x}_{2}^{2}, \\
& \hat{\varphi}_{4,3}=3 \hat{x}_{1} \hat{x}_{2}^{2}-2 \hat{x}_{1} \hat{x}_{2}^{3}-4 \hat{x}_{1}^{2} \hat{x}_{2}^{2}, \\
& \hat{\varphi}_{4,4}=-\hat{x}_{1} \hat{x}_{2}^{2}+\hat{x}_{1} \hat{x}_{2}^{3}+\hat{x}_{1}^{2} \hat{x}_{2}^{2} .
\end{aligned}
$$

Let $\hat{v} \in P_{\hat{e}}^{2}$ be an arbitrary function. Along the side $\hat{a}_{1} \hat{a}_{2}$, it is a polynomial of degree 3 in $\hat{x}_{1}$. Together with the derivative $\partial_{1} \hat{v}$, it is uniquely determined by the values $\hat{v}\left(\hat{a}_{1}\right), \hat{v}\left(\hat{a}_{2}\right), \partial_{1} \hat{v}\left(\hat{a}_{1}\right)$, and $\partial_{1} \hat{v}\left(\hat{a}_{2}\right)$ of nodal parameters. In addition, the derivative $\partial_{2} \hat{v}$ along $\hat{a}_{1} \hat{a}_{2}$ is a polynomial of degree 3 in $\hat{x}_{1}$ as well and is uniquely determined by the values $\partial_{2} \hat{v}\left(\hat{a}_{1}\right), \partial_{2} \hat{v}\left(\hat{a}_{2}\right), \partial_{1,2} \hat{v}\left(\hat{a}_{1}\right)$, and $\partial_{1,2} \hat{v}\left(\hat{a}_{2}\right)$ of nodal parameters. On the side $\hat{a}_{1} \hat{a}_{4}$ similar statements are valid within the replacement of $\hat{x}_{1}$ by $\hat{x}_{2}$.

Thus, on the sides $\hat{a}_{1} \hat{a}_{2}$ and $\hat{a}_{1} \hat{a}_{4}$ the values of a function of $P_{\hat{e}}^{2}$ and its first-order partial derivatives are uniquely determined by the values of nodal parameters of $\Sigma_{\hat{e}}^{2}$ at the nodes on the corresponding side.

On the side $\hat{a}_{2} \hat{a}_{4}$ a similar property does not hold. Generally speaking, the element $\left(\hat{e}, P_{\hat{e}}^{2}, \Sigma_{\hat{e}}^{2}\right)$ is not of the class $C^{1}$. This follows from the fact that the firstorder partial derivatives of the basis functions related to the node $\hat{a}_{1}$ do not vanish on the side $\hat{a}_{2} \hat{a}_{4}$. However, further we assume that the side of any triangular element of $\mathfrak{J}_{h}$ being the image of the hypotenuse $\hat{a}_{2} \hat{a}_{4}$ is a part of the boundary and can not be a common side of two meshes. Hence, this feature has no influence on interelemental continuity inside the domain.

To check the interpolation properties, we use the usual notations for Sobolev spaces. Here $H^{0}(\Omega)$ is the Hilbert space of functions, Lebesgue measurable on $\Omega$, equipped with the inner product

$$
(u, v)_{\Omega}=\int_{\Omega} u v \mathrm{~d} \Omega, \quad u, v \in H^{0}(\Omega),
$$

and the finite norm

$$
|u|_{0, \Omega}=(u, u)_{\Omega}^{1 / 2}, \quad u \in H^{0}(\Omega) .
$$

For integer nonnegative $k, H^{k}(\Omega)$ is the Hilbert space of functions $u \in H^{0}(\Omega)$ whose weak derivatives up to order $k$ inclusive belong to $H^{0}(\Omega)$. The norm in this space is defined by the formula

$$
\|u\|_{k, \Omega}=\left(\sum_{0 \leq s+r \leq k}\left|\frac{\partial^{s+r} u}{\partial x_{1}^{s} \partial x_{2}^{r}}\right|_{0, \Omega}^{2}\right)^{1 / 2} .
$$

We also use the seminorm

$$
|u|_{k, \Omega}=\left(\sum_{s+r=k}\left|\frac{\partial^{s+r} u}{\partial x_{1}^{s} \partial x_{2}^{r}}\right|_{0, \Omega}^{2}\right)^{1 / 2}, \quad u \in H^{k}(\Omega) .
$$

Let $\hat{u}$ be an arbitrary function of $H^{4}(\hat{e})$. By a Sobolev embedding theorem, $H^{4}(\hat{e})$ is continuously embedded into $C^{2}(\hat{e})$ [20], hence, $\hat{u} \in C^{2}(\hat{e})$. Thus, we can construct its interpolant $\hat{u}_{I} \in P_{\hat{e}}^{2}$. Denote by $m_{i}, i=1, \cdots, 4$, the number of degrees of freedom related to a node $\hat{a}_{i}$. We have

$$
\hat{u}_{I}^{1}\left(\hat{x}_{1}, \hat{x}_{2}\right)=\sum_{i=1}^{4} \sum_{j=1}^{m_{i}} \hat{\psi}_{i, j}(\hat{u}) \hat{\varphi}_{i, j}\left(\hat{x}_{1}, \hat{x}_{2}\right) .
$$

Theorem 1. Let $\hat{u} \in H^{4}(\hat{e})$. Then for any integer $m \leq 4$ we have the estimate

$$
\left|\hat{u}-\hat{u}_{I}\right|_{m, \hat{e}} \leq c_{1}|\hat{u}|_{4, \hat{e}}
$$

with constant $c_{1}$ independent of $\hat{u}$ (and $h$ ). 
Proof. The maximal order of partial derivatives equals 2 in the definition of the set $\Sigma_{\hat{e}}^{2}$. As mentioned above, the space $H^{4}(\hat{e})$ is embedded into $C^{2}(\hat{e})$. Besides, from (3) it follows that $P_{\hat{e}}^{2} \supset P_{3}(\hat{e})$ where $P_{3}(\hat{e})$ is the space of polynomials of degree no more than 3 .

Thus, all the hypotheses of the Theorem 3.1.5 in [12] are fulfilled, which implies the estimate (6).

\section{Combination of Rectangular and Triangular Elements}

Let $e \in \mathfrak{I}_{h}$ be an arbitrary triangular element (Figure 3) with vertices $a_{1}^{e}=\left(a_{1,1}^{e}, a_{1,2}^{e}\right), \quad a_{2}^{e}=\left(a_{2,1}^{e}, a_{1,2}^{e}\right)$, $a_{4}^{e}=\left(a_{1,1}^{e}, a_{4,2}^{e}\right)$ and side lengths $h_{1}^{e}=a_{2,1}^{e}-a_{1,1}^{e}$, $h_{2}^{e}=a_{4,2}^{e}-a_{1,2}^{e}$. The affine mapping $\left(x_{1}, x_{2}\right)=f_{e}\left(\hat{x}_{1}, \hat{x}_{2}\right)$, that maps the «reference» element $\hat{e}$ into $e$, has the form

$$
x_{1}=h_{1}^{e} \hat{x}_{1}+a_{1,1}^{e}, \quad x_{2}=h_{2}^{e} \hat{x}_{2}+a_{1,2}^{e} .
$$

We specify the space $P_{e}^{2}$ of functions and the set $\Sigma_{e}^{2}$ of degrees of freedom as follows:

$$
\begin{aligned}
& P_{e}^{2}= \operatorname{span}\left\{1, x_{1}, x_{2}, x_{1}^{2}, x_{2}^{2}, x_{1} x_{2}, x_{1}^{3}, x_{2}^{3},\right. \\
&\left.x_{1}^{2} x_{2}, x_{1} x_{2}^{2}, x_{1}^{2} x_{2}^{2}, x_{1}^{3} x_{2}, x_{1} x_{2}^{3}\right\}, \\
& \Sigma_{e}^{2}=\left\{\psi_{i, 1}^{e}(p)=p\left(a_{i}^{e}\right), i=1, \cdots, 4, \psi_{i, 2}^{e}(p)=\partial_{1} p\left(a_{i}^{e}\right),\right. \\
&\left.\psi_{i, 3}^{e}(p)=\partial_{2} p\left(a_{i}^{e}\right), \psi_{i, 4}^{e}(p)=\partial_{1,2} p\left(a_{i}^{e}\right), i=1,2,4\right\} .
\end{aligned}
$$

The Lagrange basis in $P_{e}^{2}$ consists of the functions $\varphi_{i, j}^{e}(x)$, where $j=1, \cdots, 4$ for $i=1,2,4$ and $j=1$ for $i=$ 3 being the images of the basis (5) under the mapping (7) and satisfying the condition

$$
\psi_{i, j}^{e}\left(\varphi_{k, l}^{e}\right)=\delta_{i, k} \delta_{j, l} .
$$

Thus, the triple $\left(e, P_{e}^{2}, \Sigma_{e}^{2}\right)$ is a finite element that is affinely equivalent to the «reference» element $\left(\hat{e}, P_{\hat{e}}^{2}, \Sigma_{\hat{e}}^{2}\right)$ [12].

Now denote the set of all nodes of the elements $e \in \mathfrak{J}_{h}$ by $\Omega_{h}$ and number them from 1 to $s$. With each node $y_{k} \in \Omega_{h}$ we associate the number $m_{k}$ equal

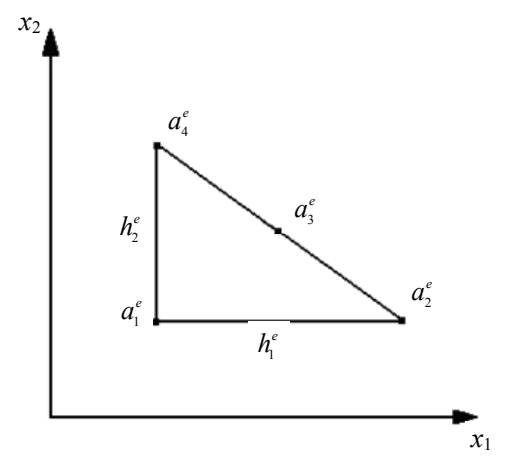

Figure 3. A triangular cell $e \in \mathfrak{I}_{h}$. to the number of degrees of freedom related to this node. Observe that $m_{k}=1$ is occurred when the node $y_{k}$ is the midpoint of the side of a triangular element being a part of the boundary $\Gamma$ and $m_{k}=4$ for all remaining nodes of $\Omega_{h}$.

This is the global numbering of nodes. We also introduce the local numbering. The couple $(i, e)$ is assumed to be a local number of the node $a_{i}^{e}$ of an element $e \in \mathfrak{I}_{h}$. To any local number $(i, e)$ there corresponds one and only one global number $k$, hence, we can introduce the function $q(i, e)$ so that $k=q(i, e)$. In addition, for an element $e \in \mathfrak{I}_{h}$ we denote the local analogue of the parameter $m_{k}$ by $m_{i}^{e}$, i.e., $m_{k}=m_{i}^{e}$ for $k=q(i, e)$.

At each node $y_{k} \in \Omega_{h}, k=1, \cdots, s$, we specify $m_{k}$ numbers $v_{k, j}, j=1, \cdots, m_{k}$. Construct the function $v_{h}$ defined on $\Omega$ such that $\forall e \in \mathfrak{I}_{h}$

$$
\left.v_{h}\right|_{e}=\sum_{i=1}^{4} \sum_{j=1}^{m_{i}^{e}} v_{q(i, e), j} \varphi_{i, j}^{e} .
$$

By the construction, the function $v_{h}$ is uniquely defined on each $e \in \mathfrak{I}_{h}$. Put

$$
P_{e}= \begin{cases}P_{e}^{1} & \text { if } e \in \mathfrak{J}_{h} \text { is a rectangle, } \\ P_{e}^{2} & \text { if } e \in \mathfrak{J}_{h} \text { is a triangle. }\end{cases}
$$

Then $\left.v_{h}\right|_{e} \in P_{e}$ for any $e \in \mathfrak{I}_{h}$.

Lemma 2. The function $v_{h}$ defined by the relation (10) belongs to $C^{1}(\bar{\Omega})$.

Proof. The BFS-rectangles are elements of the class $C^{1}$, i.e., the function $v_{h}$ and its first-order derivatives are continuous on the sides common for two elements of this type [10].

Now let $e_{\alpha} \in \mathfrak{I}_{h}$ be an arbitrary triangular cell and $e_{\beta} \in \mathfrak{I}_{h}$ be a rectangular cell that has a common side $\gamma$ with $e_{\alpha}$ (Figure 4). Because of construction, the values of the nodal parameters of the functions $\left.v_{h}\right|_{e_{\alpha}}$ and $\left.v_{h}\right|_{e_{\beta}}$ on $\gamma$ are equal. In addition, the traces of these functions and their first-order derivatives with respect to $x_{1}$ on $\gamma$ are polynomials of degree 3 in $x_{2}$ and are uniquely defined by the sets of nodal parameters related to the side $\gamma$. Hence, the functions $\left.v_{h}\right|_{e_{\alpha}}$ and $\left.v_{h}\right|_{e_{\beta}}$ coincide on $\gamma$ along with their first-order derivatives.

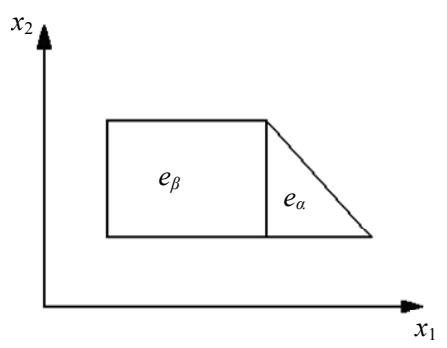

Figure 4. Two neighbouring elements of different types. 
Corollary 1. $v_{h} \in H^{2}(\Omega)[12,21]$.

Thus, we can define the finite element space as follows:

$$
V_{h}=\left\{v_{h} \in C^{1}(\bar{\Omega}):\left.v_{h}\right|_{e} \in P_{e} \quad \forall e \in \mathfrak{I}_{h}\right\} \subset H^{2}(\Omega) .
$$

Let $u \in C^{2}(\bar{\Omega})$. Define its interpolant $u_{I} \in V_{h}$ in the following way:

$$
\left.u_{I}\left(x_{1}, x_{2}\right)\right|_{e}=\sum_{i=1}^{4} \sum_{j=1}^{m_{i}^{e}} \psi_{i, j}^{e}(u) \varphi_{i, j}^{e}\left(x_{1}, x_{2}\right) \quad \forall e \in \mathfrak{I}_{h} .
$$

With the help of the Theorem 1, the following estimate can be proved in the usual way (see, for instance, $[12,14]$ ).

Theorem 2. Assume that $u \in H^{4}(\Omega)$. And let $u_{I} \in V_{h}$ be its interpolant defined by (11). Then

$$
\left\|u-u_{I}\right\|_{m, \Omega} \leq c_{2} h^{4-m}\|u\|_{4, \Omega}, \quad 0 \leq m \leq 2 .
$$

Here and later constants $c_{i}$ are independent of $h$ and $u$.

\section{Numerical Example}

We illustrate properties of the proposed finite elements by the following example. Let $\Omega$ be a right triangle with unit catheti (see Figure 5) and a boundary $\Gamma$. Consider the problem

$$
\begin{array}{ll}
-\Delta u=f & \text { in } \Omega, \\
u=0 & \text { on } \Gamma
\end{array}
$$

with the right-hand side

$$
f(x, y)=2(x+y) \cos (1-x-y)+2 x y \sin (1-x-y) .
$$

It has the exact solution

$$
u(x, y)=x y \sin (1-x-y) \text {. }
$$

Subdivide the domain $\Omega$ into elementary squares (with triangles adjacent to the hupotenuse) by drawing two families of parallel straight lines $x_{i}=i h, i=1, \cdots, n-1$, and $y_{j}=j h, j=1, \cdots, n-1$ with mesh size $h=1 / n$.

To compare accuracy with decreasing mesh size, we construct a system of linear algebraic equations of the finite element method with the BFS-elements on the elementary squares and the proposed elements on the

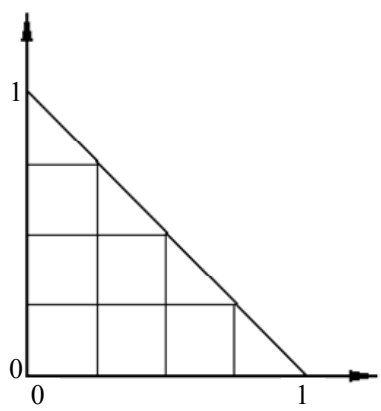

Figure 5. Domain $\Omega$ with initial triangulation. triangles for $n=4$ and $n=8$. Since the exact solution is known, the difference $u-u_{h}$ between exact and approximate solutions can be expressed in the explicit form. As a result, we have the following accuracy.

Theoretically, in the finite element method we have the estimate $[12,14]$

$$
\left\|u-u^{h}\right\|_{m, \Omega} \leq c_{3}\left\|u-u_{I}\right\|_{m, \Omega}, \quad m=1,2 .
$$

Combining it with the estimate in Theorem 2, we arrive at the following error estimate for an approximate solution:

$$
\left\|u-u^{h}\right\|_{m, \Omega} \leq c_{4} h^{4-m}\|u\|_{4, \Omega}, \quad m=1,2
$$

Comparing the last two results in Table 1, observe that they are close to the asymptotic values 4 and 3 , respectively.

\section{Summary and Further Implementations}

Thus, the use of the proposed triangular finite elements only near a boundary extends the field of application of the BFS-elements at least for second order equations. In its turn, an approximate solution is of the class $H^{2}(\Omega)$ enabling one to calculate a residual directly and considerably simplifies a posteriori accuracy estimates for an approximate solution.

In principle, to achieve the same order accuracy, instead of the BFS-elements, one can use the Lagrange bicubic elements on rectangles and the Lagrange elements of degree three on triangles. But in this case a general advantage of Hermite finite elements in com-

\begin{tabular}{|c|c|c|}
\hline$h$ & $\delta_{h}=\left\|u-u^{h}\right\|_{0, \Omega}$ & $\sigma_{h}=\left\|u-u^{h}\right\|_{1, \Omega}$ \\
\hline 0.25 & $2.661 \times 10^{-6}$ & $8.664 \times 10^{-5}$ \\
\hline 0.125 & $1.724 \times 10^{-7}$ & $1.124 \times 10^{-5}$ \\
\hline$\delta_{2 h} / \delta_{h}$ & $\log _{2}\left(\delta_{2 h} / \delta_{h}\right)$ & $\log _{2}\left(\sigma_{2 h} / \sigma_{h}\right)$ \\
\hline 15.43 & 7.71 & 2.95 \\
\hline
\end{tabular}
parison with Lagrange ones makes itself evident in the number of unknowns of a discrete system. In particular, Table 2 shows the number of degrees of freedom for an approximate solution which is equal to the number of unknowns and the number of equations in a discrete system of linear algebraic equations for the example from the above section.

Table 1. Accuracy of an approximate solution.

Table 2. The numbers of degrees of freedom for an approximate solution.

\begin{tabular}{ccc}
\hline$h$ & Hermite elements & Lagrange elements \\
\hline 0.25 & 33 & 55 \\
0.125 & 129 & 253 \\
\hline
\end{tabular}




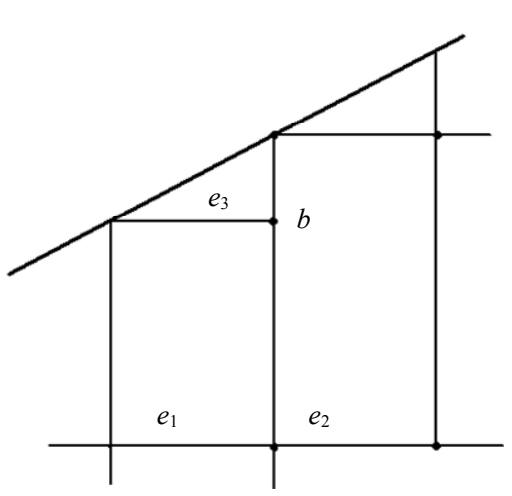

Figure 6. A "hanging" node $b$.

Observing a considerable gain in the number of unknowns, with the further refinement of triangulation, the number of unknowns (and equations) asymptotically tends to the ratio of 4:9 in favour of Hermite elements.

Now we notice an apparent inconvenience and show a way to overcome it that is also useful for nonconforming grid refinement. The triangulation shown in Figure 1 is constructed by adjusting cells of a rectangular grid in the $x_{1}$ - and $x_{2}$-directions. It imposes restrictions on the ratio of steps for a rectangular grid inside a domain. We show that the proposed triangular elements are sufficient to construct a conforming interpolant, generally speaking, on an "uncoordinated" grid without restriction on the ratio between steps.

Considering a typical case shown in Figure 6, the node $b$ is a so-called "hanging" node. At the algorithmic level, the challenge is solved as follows. For the degrees of freedom at the node $b$, instead of the corresponding variational equations for an approximate solution $u^{h}$, we write 4 linear algebraic equalities which express the quantities $u^{h}(b), \partial_{1} u^{h}(b), \partial_{2} u^{h}(b)$, and $\partial_{1,2} u^{h}(b)$ in terms of 16 degrees of freedom of the neighbouring rectangle $e_{2}$. This trick ensures interelemental continuous differentiability of an interpolant between $e_{1}, e_{2}$, and $e_{3}$. Thus in this case one again may implement proposed pair of elements in the frame of the conforming finite element method with estimate (12).

\section{REFERENCES}

[1] J. H. Argyris, I. Fried and D. W. Scharpf, "The TUBA Family of Plate Elements for the Matrix Displacement Method," Journal of the Royal Aeronautical Society, Vol. 72, No. 692, 1968, pp. 701-709.

[2] K. Bell, "A Refined Triangular Plate Bending Element", International Journal of Numerical Methods in Engineering, Vol. 1, No. 1, 1969, pp. 101-122. http://dx.doi.org/10.1002/nme.1620010108

[3] J. Morgan and L. R. Scott, "A Nodal Basis for $C^{1}$ Piecewise Polynomials of Degree n," Mathematics of Computation, Vol. 29, No. 131, 1975, pp. 736-740.

[4] R. W. Clough and J. L. Tocher, "Finite Element Stiffness
Matrices for Analysis of Plates in Bending," Proceedings of the Conference on Matrix Methods in Structural Mechanics, Wright-Patterson Air Force Base, Ohio, October 1965, pp. 515-545.

[5] P. Percell, "On Cubic and Quartic Clough-Tocher Finite Elements," SIAM Journal on Numerical Analysis, Vol. 13, No. 1, 1976, pp. 100-103. http://dx.doi.org/10.1137/0713011

[6] J. Douglas Jr., T. Dupont, P. Percell and R. Scott, "A Family of $C^{1}$ Finite Elements with Optimal Approximation Properties for Various Galerkin Methods for 2nd and 4th Order Problems", RAIRO Analise Numérique, Vol. 13, No. 3, 1979, pp. 227-255.

[7] M. J. D. Powell and M. A. Sabin, "Piecewise Quadratic Approximations on Triangles," ACM Transactions on Mathematical Software, Vol. 3-4, No. 4, 1977, pp. 316325. http://dx.doi.org/10.1145/355759.355761

[8] B. Fraeijs de Veubeke, "Bending and Stretching of Plates", Proceedings of the Conference on Matrix Methods in Structural Mechanics, Wright-Patterson Air Force Base, Ohio, October 1965, pp. 863-886.

[9] B. Fraeijs de Veubeke, "A Conforming Finite Element for Plate Bending," In: J. C. Zienkievicz and G. S. Holister, Eds., Stree Analysis, Wiley, New York, 1965, pp. 145197.

[10] F. K. Bogner, R. L. Fox and L. A. Schmit, "The Generation of Interelement Compatible Stiffness and Mass Matrices by the Use of Interpolation Formulas," Proceedings of the Conference on Matrix Methods in Structural Mechanics, Wright-Patterson Air Force Base, Ohio, October 1965, pp. 397-444.

[11] S. Zhang, "On the full $\mathrm{C}_{1}-\mathrm{Q}_{\mathrm{k}}$ Finite Element Spaces on Rectangles and Cuboids," Advances in Applied Mathematics and Mechanics, Vol. 2, No. 6, 2010, pp. 701-721.

[12] P. G. Ciarlet, "The Finite Element Method for Elliptic Problems," North-Holland, Amsterdam, 1978.

[13] Z. C. Li and N. Yan, "New Error Estimates of Bi-Cubic Hermite Finite Element Methods for Biharmonic Equations," Journal of Computational and Applied Mathematics, Vol. 142, No. 2, 2002, pp. 251-285. http://dx.doi.org/10.1016/S0377-0427(01)00494-0

[14] S. C. Brenner and L. R. Scott, "The Mathematical Theory of Finite Element Methods," Springer-Verlag, New York, 1994. http://dx.doi.org/10.1007/978-1-4757-4338-8

[15] B. M. Irons, "A Conforming Quadratic Triangular Element for Plate Bending," International Journal for $\mathrm{Nu}$ merical Methods in Engineering, Vol. 1, No. 1, 1969, pp. 101-122.

[16] D. L. Logan, "A First Course of Finite Element Method," SI Edition, 2011.

[17] J. Blaauwendraad, "Plates and FEM: Surprises and Pitfalls," Springer, New York, 2010. http://dx.doi.org/10.1007/978-90-481-3596-7

[18] J. Zhao, "Convergence of V- and F-cycle Multigrid Methods for Biharmonic Problem Using the HsiehClough-Tocher Element," Numerical Methods for Partial Differential Equations, Vol. 21, No. 3, 2005, pp. 451-471. http://dx.doi.org/10.1002/num.20048 
[19] J. Petera and J. F. T. Pittman, "Isoparametric Hermite elements," Intenational Journal for Numerical Methods in Engineering, Vol. 37, No. 20, 1994, pp. 3480-3519.

[20] R. A. Adams and J. J. F. Fournier, "Sobolev Spaces,"
Academic Press, New York, 2003.

[21] Z. Chen and H. Wu, "Selected Topics in Finite Element Methods," Science Press, Beijing, 2010. 